\title{
Quantifying co-cultured cell phenotypes in high-throughput using pixel-based classification
}

\author{
David J. Logana, Jing Shan ${ }^{b}$, Sangeeta N. Bhatia ${ }^{\text {abcdefg }}$, and Anne E. Carpenter \\ a The Broad Institute of MIT and Harvard \\ 415 Main Street, Cambridge, MA 02142 \\ ${ }^{\mathrm{b}}$ Harvard-MIT Division of Health Sciences and Technology, MIT \\ E25-518, 77 Massachusetts Ave, Cambridge, MA 02139 \\ 'Department of Medicine, Brigham and Women's Hospital \\ 75 Francis St, Boston, MA 02115 \\ ${ }^{d}$ Institute for Medical Engineering and Science, MIT \\ E25-330, 77 Massachusetts Ave, Cambridge, MA 02139 \\ e Department of Electrical Engineering and Computer Science, MIT \\ 38-401, 77 Massachusetts Ave, Cambridge, MA 02139 \\ ${ }^{\mathrm{f}}$ David H. Koch Institute for Integrative Cancer Research, MIT \\ 76-158, 77 Massachusetts Avenue, Cambridge, MA 02139 \\ 9 Howard Hughes Medical Institute \\ 4000 Jones Bridge Road, Chevy Chase, MD 20815-6789
}

Email Addresses:

David J. Logan: dlogan@broadinstitute.org Jing Shan: js8686@mit.edu Sangeeta N. Bhatia: sbhatia@mit.edu Anne E. Carpenter: anne@broadinstitute.org

\author{
Please address correspondence to: \\ Anne E. Carpenter, Ph.D. \\ The Broad Institute of MIT and Harvard \\ 415 Main Street \\ Cambridge, MA 02142 \\ Tel: $617-714-7750$ \\ Fax: 617-714-8957 \\ anne@broadinstitute.org
}




\begin{abstract}
Biologists increasingly use co-culture systems in which two or more cell types are grown in cell culture together in order to better model cells' native microenvironments. Co-cultures are often required for cell survival or proliferation, or to maintain physiological functioning in vitro. Having two cell types co-exist in culture, however, poses several challenges, including difficulties distinguishing the two populations during analysis using automated image analysis algorithms. We previously analyzed co-cultured primary human hepatocytes and mouse fibroblasts in a high-throughput image-based chemical screen, using a combination of segmentation, measurement, and subsequent machine learning to score each cell as hepatocyte or fibroblast. While this approach was successful in counting hepatocytes for primary screening, segmentation of the fibroblast nuclei was less accurate. Here, we present an improved approach that more accurately identifies both cell types. Pixel-based machine learning (using the software ilastik) is used to seed segmentation of each cell type individually (using the software CellProfiler). This streamlined and accurate workflow can be carried out using freely available and open source software.
\end{abstract}

\title{
Key Words
}

High content screening; image analysis; open-source software; assay development; co-culture; hepatocytes

\section{Introduction}

Biologists increasingly use whole organisms and co-culture systems in an effort to create more physiological experimental systems. The mechanisms by which cells respond to their local microenvironment and determine appropriate cellular functions is complex and poorly understood. In many cases, co-culture systems are required for a particular cell type to proliferate or to maintain viability and physiological functioning in vitro. These increasingly complex model systems also more faithfully represent the native cellular microenvironment. Co-culture systems provide a valuable model for dissecting the mechanisms of cell signaling, whether by diffusible small molecules and exosomes, or by contact through cell-cell interactions and extracellular matrix deposition. Co-culture systems are also being used to study cellular biomechanics in cell migration [1], hepatocyte functions (transporters, metabolism, regeneration, infection, toxicity, extracellular matrix, and tissue structure/function relationships, development, and size control) [2], embryogenesis (growth, development, autocrine and paracrine regulation) [3], cartilage (physiology, homeostasis, repair and regeneration) [4], cancer (growth, invasion, metastasis, and differentiation) [5], and stem cells (differentiation and development) [6], among others. 
Automated image analysis is desperately needed for co-culture systems. Microscopy is a powerful means to separate the cells into virtual mono-cultures for analysis purposes and can be quantitative if suitable algorithms exist. Identifying cells of one particular cell type is typically feasible using existing algorithms; however, these analyses can falter when faced with a dense mixture of two cell types of distinct morphology. Properly identifying mixtures of two object types is a challenging computational problem: most algorithms depend on building a model of a single object type. As yet, no model-based segmentation (object delineation) algorithms have been demonstrated to be generally useful for co-culture systems lacking specific labels. Until now, each cell type must typically be segmented separately in co-culture experiments, requiring laborious individual algorithmic parameter settings or an object-based classification step that can distinguish each object type (using e.g. size, texture, or intensity). It would be preferable to simplify the steps of distinguishing and segmenting the cells. Solutions are needed to render the new co-culture systems tractable to automated image analysis, a tool that has become indispensable throughout biology.

We previously developed a high-throughput, image-based screening platform for primary human hepatocytes co-cultured with fibroblasts, together with an informatics workflow to process the resulting images [7]. We used it to identify small molecules that induced functional proliferation of primary human hepatocytes, with an ultimate goal of generating renewable and functional cell sources for liver research and the treatment of liver diseases. As such, the informatics workflow was optimized for counting hepatocytes; its accuracy for identifying and counting fibroblasts was not ideal. This drawback consequently prevented in-depth analyses of any statistical correlations that required accurate fibroblast cell identification in addition to hepatocyte counts.

Here, we present a novel informatics workflow that is simplified and capable of accurate counting of multiple, fluorescent morphologies. It overcomes many of the limitations of the prior workflow, which relied on segmentation (relatively accurate for hepatocytes, but with fibroblasts often over-segmented) followed by machine learning to classify hepatocytes versus fibroblasts (or portions thereof). Here, we accurately segment and count both cell types by using pixel-based machine learning [8,9] followed by model-based segmentation (tuned to hepatocyte and fibroblast morphology separately) and counting. We demonstrate that this workflow is more user-friendly, and provides improved accuracy.

\section{Materials and Methods}

\subsection{Cell Culture}

Details of the cell culture methods have been previously published [7]. Briefly, J2-3T3 fibroblasts were plated on collagen-coated 384-well plates at a density of 8,000 cells per well. After 48 hours, primary human hepatocytes were plated onto the fibroblasts at densities ranging from 4000 to 9500 cells per well; as a result, fibroblasts generally outnumber hepatocytes in the final images. Cells were fixed and stained with Hoechst 33342 to visualize the nuclei. 


\subsection{Microscopy and Image Acquisition}

Details of the microscopy and image acquisition methods have been previously published [7]. Here, one 384-well plate was populated with alternating wells empty, resulting in 96 wells of samples. Nine sites per well were robotically imaged (Molecular Devices, Inc.) at 20x objective magnification, which was sufficient to visualize differences between the cell types. Note that the sites imaged did not span the entire well, so the cell counts do not sum to the numbers of cells seeded (plus their daughter cells). For the analysis presented here, two columns of wells from the plate were analyzed: a low hepatocyte count column (4000 hepatocytes seeded per well) of 8 wells, and a high hepatocyte count column (9500 hepatocytes seeded per well) of 8 wells, totaling 144 images.

\subsection{Image Analysis}

All CellProfiler pipelines and detailed settings to reproduce the image analysis procedures are available here: http://www.cellprofiler.org/published_pipelines.shtml. The image processing for the two workflows presented involves three open-source software packages (detailed in the "Computational resources" section below): CellProfiler ([10], http://cellprofiler.org), CellProfiler Analyst ([11], http://cellprofiler.org), and ilastik ([9], http://ilastik.org). Subsequent sections provide a narrative overview of the processing steps.

\subsubsection{Illumination Correction}

To account for systematic bias due to non-homogeneous illumination across the image field, all images were illumination-corrected [12]. A dedicated CellProfiler pipeline loaded all the images from the plate and averaged them. This average image was then smoothed using a median filter (width: 300 pixels) and saved. The smoothed image, called the illumination function, is subsequently loaded into the main CellProfiler pipelines (described below, sections 2.3.2 and 2.3.3), and each raw image is then divided by the illumination function to achieve a set of illumination-corrected images. These images were saved, and used as inputs for both the previous and new workflows.

\subsubsection{Previous Workflow}

Details of the previous workflow have been previously published [7]. Briefly, illumination corrected images (section 2.3.1) were loaded into CellProfiler using the LoadData module. All nuclei were segmented using three-class Otsu thresholding, with intensity-based declumping. Multiple measurements were made on the resulting segmented objects in order to facilitate the object-based machine learning: texture at scales of 1 and 3 pixels, adjacency metrics up to 25 
pixels from each nucleus object, intensity-based statistics within each object, morphology/shape based features, and the radial distribution of intensity within each object.

Further, because punctate nuclear spots are a prominent feature of murine fibroblasts, these features were analyzed separately. The puncta were enhanced with a tophat filter with a 9 pixel width. Puncta were segmented with CellProfiler's RobustBackground thresholding method, and declumped using local intensity peaks' maxima. Intensities and shape features were measured for each spot, and they were counted relative to their parent nucleus.

CellProfiler Analyst's Classifier tool [11] was used to load the database tables created and populated with all the measurements made with CellProfiler. Using 60 "rules", i.e., regression stumps, from a GentleBoosting algorithm, objects were classified as hepatocyte, fibroblast, or debris. Approximately 329 nuclei (randomly selected from 238 images) were used for training in CellProfiler Analyst. Care was taken to exclude the test set of images/wells (used for later analysis) from the training set. All objects were then scored and counted on a per-well basis.

\subsubsection{New Workflow}

Approximately 60 nuclei plus 4 regions deemed debris (from 4 images) were used for training in ilastik. Hepatocyte and fibroblast nuclei were manually labeled separately with the paintbrush tool, as well as debris and background pixel classes. Training was iterative, adding new pixel classification until the probability maps were stable and adequately distinguished the object types. The ilastik classifier was exported as an HDF5 file.

A CellProfiler pipeline loaded the illumination corrected images, then imported the ilastik classifier file as an HDF5 file and applied it to each image. The hepatocyte and fibroblast probability maps were smoothed with a Gaussian filter of width 7 pixels (debris and background classes were not analyzed further). Hepatocyte and fibroblast objects were segmented by simply thresholding the probability maps with a manual threshold of 0.5 (i.e. $>50 \%$ probability), and declumping based on shape using the distance transform of the objects. All objects were then counted on a per-well basis.

\subsection{Ground truth comparison}

A small set of random images not in either training set were manually labeled with 3 colors, marking fibroblasts, hepatocytes, and other objects (mostly debris or bright dying cells). This set included 1414 total objects (981 fib, 408 hep, 25 debris/other) in five images. These

manually-labeled images were loaded into our old and new CellProfiler pipelines and compared 
against the old and new method's object classifications. The ground truth pipelines are available with the other pipelines (see section 2.5).

\subsection{Computational resources}

Both image processing workflows were tested on a desktop Windows 7 workstation (16 GB RAM) and CellProfiler pipelines were submitted for processing on a Linux cluster.

Software versions used were CellProfiler version 2.1.2 v2015_08_05, CellProfiler Analyst 2.0 v2014_04_01, and ilastik version 0.5.12; links to Windows binary versions of these are provided here: http://www.cellprofiler.org/published pipelines.shtml. Note that newer versions of ilastik exist but are not currently supported by CellProfiler. Note also that the training step in the new workflow can be carried out using ilastik on Mac OS X, but CellProfiler's ClassifyPixels currently is supported on Windows only.

\section{Results and Discussion}

We developed a novel, streamlined informatics workflow to process co-culture images of hepatocytes and fibroblasts (Figure 1) based on pixel-based machine learning followed by segmentation (Figure 2B). This workflow begins with the researcher marking a few regions as belonging to the classes "hepatocyte", "fibroblast", and "background" within a small set of fluorescent images taken from the entire experiment (Figure 2B, top). This labeling is done with a paintbrush-style tool in the open-source software tool ilastik [9], which is very simple to use and requires $\sim 2$ hours for a typical co-culture data set. Pixel classifiers, as in ilastik, aim to learn to distinguish whether each pixel belongs to a specific object type or background, using not just the intensity information of that pixel but also intensity information from local pixel neighborhoods [8].

One advantage of using ilastik for pixel-based machine learning is that the researcher need not understand the details of the machine learning process; the researcher simply marks regions with the paintbrush iteratively until the results appear sufficiently accurate. Behind the scenes, the labeled pixels form the training set for a pixel-based random-forest classifier. The classifier takes intensity, texture, edge, and orientation categories of features at multiple scales (3 to 61 pixels), as input. Here, we chose all features and scales, as this broad selection was not prohibitive computationally (e.g. less than 60 seconds to train on 3 images) but the number of features could reasonably be reduced depending on the image assay characteristics. This classifier is refined iteratively until the researcher is qualitatively satisfied that the output probability maps show adequate assignment of the pixels to each of the classes of cell type across a small subset of test images. ilastik also reports the 'out-of-bag (oob) error' which is an 
unbiased estimate of the test set error for random forests (mean oob $=0.01$ for 3 individual images trained and tested).

The resulting pixel-based classifier is then exported and loaded into the open-source software CellProfiler $[10,13]$ via the ClassifyPixels module. This new module is designed to accept ilastik classifiers as input and apply them to all images in the experiment. This process produces separate probability maps for hepatocytes and for fibroblasts, where the relative brightness of a pixel indicates the likelihood of that pixel belonging to the cell type of interest (Figure 2B, middle). In the context of a CellProfiler pipeline, the ClassifyPixels module thereby serves to pre-process the input image for downstream segmentation, counting, and measurement of cells. The resulting probability map images of each cell type are then smoothed with a small Gaussian filter ( 7 pixels wide) to prevent over-segmentation, justified by inspection that nuclei are generally at least 7 pixels wide and convex. The objects are segmented and counted using these smoothed probability maps in the remainder of the pipeline (Figure 2B, bottom), which can optionally include measurement of morphological features of both types of nuclei.

The previous workflow (Figure 2A) also used machine learning, but did so at the object level, after segmentation (Figure 2A, bottom), rather than at the pixel level, prior to segmentation (Figure 2B, top). This restructuring led to a number of improvements, detailed below.

The training step involving user interaction is less time-consuming in the new method. In the previous workflow, we needed to train $300+$ nuclei, to ensure that we sampled across many images in the experiment while avoiding the test set, in order to adequately train the classifier. While both workflows thus involve manually training a classifier, we find that the hands-on time required to properly train the pixel-based classifier in the new workflow is much less than for training object-based classifiers in the previous workflow. The previous workflow took 4 iterations of approximately 45 minutes each to train $300+$ nuclei ( 3 hours total). The new workflow showed improved accuracy (see below) with only $64+$ nuclei/debris and background regions needing to be labeled, and in less time ( 2 hours total).

In addition, less computing time is required in the new workflow: the previous workflow relied on measuring hundreds of features of each cell for the object-based classification step, which is computationally much more time-consuming than measuring local features for pixel-based machine learning. Measuring object features is optional in the new workflow and can be limited to just those morphological features of interest for the goals of the experiment, so the analysis pipeline can process images much faster than the previous workflow. Further, if simple cell counts are the desired output, the CellProfiler pipeline is quite abbreviated and few if any measurement modules are needed, reducing time spent in image assay development and computation time. 
Moreover, because the segmentation in the previous workflow was not as accurate as the new workflow (see below), the researcher was often uncertain how to classify objects that erroneously included pixels from both cell types. In the old workflow, an initial step was used to segment both cell types simultaneously, which was difficult because of differing sizes and intensities of the nuclei classes. Accurately declumping in a single step was also problematic. With the new workflow, the researcher only needs to choose a few images and intuitively brush over nuclei to train the classifier. A coarse annotation is typically sufficient, but can be as precise as necessary to define the difficult segmentation cases, e.g. actively training adjacent nuclei results in better segmentation.

We found that the new workflow offers improved accuracy in terms of both cell counts and segmentation (Figure 3). Subjectively, we noted improved accuracy of the identification of the borders of fibroblast nuclei, especially for cases in which a fibroblast is immediately adjacent to a hepatocyte. In addition, bright "debris", which here includes bright apoptotic or mitotic nuclei, previously caused problems with the automated segmentation algorithms, even with attempts at masking bright pixels. However with ilastik, after training a "debris" class, the corresponding debris pixels have a low probability value with respect to the hepatocyte and fibroblast pixels; this streamlines the overall image analysis by eliminating the need for additional parameter tuning, and thresholding and masking steps.

We quantitatively assessed cell counts in the low-density and high-density hepatocyte wells (Figure 4). Median values across the 8 wells for each cell density show expected ratios and counts for both workflows. Note that the entire well was not imaged, so the cell counts calculated here do not sum to the numbers of cells seeded (plus their daughter cells). The variance is smaller in the new workflow, and this is reflected in an improved Z'-factor [14], commonly used for assessing assay quality (previous method $Z$ '-factor $=-1.10$, new method $Z$ '-factor $=0.16$. Fibroblasts, which were seeded at constant density throughout the plate, were also counted and found to show no significant differences between the low and high density hepatocyte conditions in either old or new workflows (Supplementary Figure S1). We further assessed other nuclear features including nuclear area and DNA content (Supplementary Figures S2 and S3). While there are differences in the area and DNA content between the old and new methods, we note that both features are quite sensitive to user-adjustable thresholding parameters. The associated parameters were not adjusted to specifically produce the same size nuclear objects; in other words, if desired this difference in size could likely be eliminated through adjustment of parameters.

To assess the accuracy of the cell type classification, classified objects from the old and new methods were compared against a set of 1414 manually annotated ground truth objects (Supplementary Figures S4 and S5). For hepatocytes, the precision increased from 0.84 to 0.94 and the recall increased from 0.64 to 0.70 (old to new). For fibroblasts, the precision increased 
from 0.85 to 0.86 , and the recall increased from 0.94 to 0.98 (old to new). To gain insight into any mislabeling biases, we tallied a truth table (Supplementary Figure S5). The old and new methods overall show similar patterns of true and false predictions. Most fibroblasts are labeled correctly, however true hepatocytes show a higher rate of mislabeling as predicted fibroblasts. This is borne out by our experience: hepatocytes' untextured nuclei are often difficult to distinguish from dim or out-of-focus fibroblast nuclei.

We suspect further improvements are compatible with the new workflow, in particular, eliminating the illumination correction step. For the purposes of consistency in this study, all images were first corrected for illumination artifacts using a separate CellProfiler pipeline as described in Materials and Methods (Section 2.3.1). In our experience, ilastik's local, pixel-based analysis appears to be inherently robust to illumination variation across an image. This flexibility can likely be attributed to ilastik's feature sets, which are based on local intensity variation and not dependent upon absolute intensities. Therefore, we anticipate the new workflow could be further simplified and accelerated by eliminating the separate illumination correction pipeline, at least for cases where subsequent measurement of intensity-based features is not part of the experimental goals.

The new approach is likely amenable to any co-culture system in which some measurable morphological or intensity differences exist between the cell types; in our experience, most visual distinctions detectable by a biologist can be classified by machine learning. This approach is therefore applicable to those situations in which clear visual distinctions exist in the co-cultured cell types, and where those distinctions can be quantified by the feature categories of ilastik. In general, as in this study, one can use nuclear morphology to distinguish any mouse cell type vs human cell type because the former has more textured chromatin [15]. Also note that ilastik feature sets can be extended by the computational biologist if needed. This should allow a broad range of co-culture systems to be analyzed with this new method.

\section{Conclusions}

In summary, we developed a workflow using pixel-based machine learning for analysis of hepatocyte/fibroblast co-culture systems that yields improved accuracy and robustness over prior object-based machine learning workflows. The new workflow is streamlined, requiring less hands-on time, less image processing expertise (due to fewer parameters to be tuned), and fewer computing resources (because morphological features of each nucleus need not be measured unless of interest in the experiment).

Because all software used in the new workflow is open-source, it is freely available as well as customizable. Therefore, the strategy presented here can be adapted and applied to a wide variety of other applications where segmentation is difficult. Potential experimental uses of this workflow include a number of other physiologically relevant model systems, especially other 
co-culture systems such as leukemia stem cells in a bone marrow microenvironment [16], parasite classification [17], and tissue samples stained with standard, non-fluorescent dyes [18]. In addition, ilastik has a plugin system that allows programmers to add problem-specific features to enrich the pixel-based classification. We have made the pipelines, example images, and all software freely available online for the scientific community to build upon.

\section{Acknowledgments}

We would like to thank the members of the Broad Institute Imaging Platform and the Bhatia Lab for helpful guidance throughout, as well as Anna Thomas for preliminary work in this area. This work was supported by a grant from the National Science Foundation (NSF CAREER DBI 1148823, to AEC) as well as the National Institutes of Health (NIH UH3 EB017103, to SNB). Dr. Bhatia is an HHMI Investigator and Merkin Institute Fellow at the Broad Institute of MIT and Harvard.

\section{References}

[1] C.-H. Yeh, S.-H. Tsai, L.-W. Wu, Y.-C. Lin, Using a co-culture microsystem for cell migration under fluid shear stress, Lab Chip. 11 (2011) 2583-2590.

[2] S.R. Khetani, S.N. Bhatia, Microscale culture of human liver cells for drug development, Nat. Biotechnol. 26 (2008) 120-126.

[3] P. Guérin, Y. Ménézo, Review: role of tubal environment in preimplantation embryogenesis: application to co-culture assays, Zygote. 19 (2011) 47-54.

[4] J. Hendriks, J. Riesle, C.A. van Blitterswijk, Co-culture in cartilage tissue engineering, J. Tissue Eng. Regen. Med. 1 (2007) 170-178.

[5] H. Dolznig, C. Rupp, C. Puri, C. Haslinger, N. Schweifer, E. Wieser, et al., Modeling colon adenocarcinomas in vitro a 3D co-culture system induces cancer-relevant pathways upon tumor cell and stromal fibroblast interaction, Am. J. Pathol. 179 (2011) 487-501.

[6] H. Salehi, K. Karbalaie, S. Razavi, S. Tanhaee, M. Nematollahi, M. Sagha, et al., Neuronal induction and regional identity by co-culture of adherent human embryonic stem cells with chicken notochords and somites, Int. J. Dev. Biol. 55 (2011) 321-326.

[7] J. Shan, R.E. Schwartz, N.T. Ross, D.J. Logan, D. Thomas, S.A. Duncan, et al., Identification of small molecules for human hepatocyte expansion and iPS differentiation, Nat. Chem. Biol. 9 (2013) 514-520.

[8] C. Sommer, D.W. Gerlich, Machine learning in cell biology - teaching computers to recognize phenotypes, J. Cell Sci. 126 (2013) 5529-5539.

[9] C. Sommer, C. Straehle, U. Kothe, F.A. Hamprecht, ilastik: Interactive learning and segmentation toolkit, in: 2011 IEEE International Symposium on Biomedical Imaging: From Nano to Macro, 2011: pp. 230-233.

[10] A. Carpenter, T. Jones, M. Lamprecht, C. Clarke, I. Kang, O. Friman, et al., CellProfiler: image analysis software for identifying and quantifying cell phenotypes, Genome Biol. 7 (2006). doi:10.1186/gb-2006-7-10-r100.

[11] T.R. Jones, A.E. Carpenter, M.R. Lamprecht, J. Moffat, S.J. Silver, J.K. Grenier, et al., Scoring diverse cellular morphologies in image-based screens with iterative feedback and 
machine learning, Proc. Natl. Acad. Sci. U. S. A. 106 (2009) 1826-1831.

[12] S. Singh, M.-A. Bray, T.R. Jones, A.E. Carpenter, Pipeline for illumination correction of images for high-throughput microscopy, J. Microsc. 256 (2014) 231-236.

[13] L. Kamentsky, T.R. Jones, A. Fraser, M.-A. Bray, D.J. Logan, K.L. Madden, et al., Improved Structure, Function, and Compatibility for CellProfiler: Modular High-Throughput Image Analysis Software, Bioinformatics. (2011). doi:10.1093/bioinformatics/btr095.

[14] J.-H. Zhang, T.D.Y. Chung, K.R. Oldenburg, A Simple Statistical Parameter for Use in Evaluation and Validation of High Throughput Screening Assays, J. Biomol. Screen. 4 (1999) 67-73.

[15] G.R. Cunha, K.D. Vanderslice, Identification in histological sections of species origin of cells from mouse, rat and human, Stain Technol. 59 (1984) 7-12.

[16] K.A. Hartwell, P.G. Miller, S. Mukherjee, A.R. Kahn, A.L. Stewart, D.J. Logan, et al., Niche-based screening identifies small-molecule inhibitors of leukemia stem cells, Nat. Chem. Biol. 9 (2013) 840-848.

[17] S. March, S. Ng, S. Velmurugan, A. Galstian, J. Shan, D.J. Logan, et al., A Microscale Human Liver Platform that Supports the Hepatic Stages of Plasmodium falciparum and vivax, Cell Host Microbe. 14 (2013) 104-115.

[18] C. Sommer, L. Fiaschi, F.A. Hamprecht, D.W. Gerlich, Learning-based mitotic cell detection in histopathological images, in: 2012 21st International Conference on Pattern Recognition (ICPR), 2012: pp. 2306-2309.

[19] M.-A. Bray, A. Carpenter, Imaging Platform, Broad Institute of MIT and Harvard, Advanced Assay Development Guidelines for Image-Based High Content Screening and Analysis, Eli Lilly \& Company and the National Center for Advancing Translational Sciences, 2013.

\section{Figure legends}

Figure 1. Example image with cell types labeled. A representative image is shown (left). Zooming in (right), example hepatocytes and fibroblasts are marked for reference. Scale bar is 20 microns.

Figure 2. A comparison of workflows for distinguishing two cell types in co-culture. (A) Our previous workflow [7] required an analysis pipeline to segment all nuclei and measure their features in CellProfiler (top), and manually-trained object-based machine learning, using CellProfiler Analyst [11] (bottom). (B) Our new workflow involves manually training a pixel-based classifier, using ilastik [9] (top) followed by an image analysis pipeline in CellProfiler that applies the classifier to create probability maps for each cell type individually (middle), then segments, counts, and (optionally) measures features for each cell of each type (bottom). For the purposes of comparison, both workflows used a pre-processing pipeline for illumination correction in CellProfiler (not shown). Scale bars are 20 microns.

Figure 3. Improved delineation of individual nuclei using pixel-based machine learning. (A) A representative raw image of a hepatocyte-fibroblast co-culture, stained with Hoechst 33342 to visualize the nuclei. (Note: intensity is log-normalized here to enhance contrast for visualization only). (B) Using the raw image for segmentation yields many nuclei improperly 
fused together or otherwise badly delineated. Note that in the full previous workflow, individual "objects" here would later be classified as hepatocyte or fibroblast using object-based machine learning. ( $C$ and $D)$ Using probability maps from pixel-based machine learning (shown in Figure 2B) for segmentation of each cell type individually yields improved delineation of adjacent nuclei, especially in cases where a hepatocyte is immediately adjacent to a fibroblast. Scale bar is 20 microns.

\section{Figure 4. Improved accuracy of counting each cell type using pixel-based machine}

learning. Boxplots comparing average hepatocyte counts per field of view for the low and high density hepatocyte conditions where 4000 and 9500 cells were initially seeded per well, respectively. (A) The previous workflow displays a clear separation between the two distributions, but with a higher standard deviation $\left(Z^{\prime}\right.$-factor $=-1.10, p$-value $\left.=3.3 \times 10^{-4}\right)$. $(B)$ The new workflow shows similar average hepatocyte counts but with less variability ( $Z^{\prime}$-factor $=0.16$, $p$-value $\left.=3.3 \times 10^{-6}\right)$. Note that higher $Z^{\prime}$-factor is better, though it should be noted that the sample sizes are suggested to be larger than those here because the statistic is sensitive to small fluctuations in data variability [19], and so if this were a true screen we would have included more control wells to push the Z' higher toward a more traditional 'screenable' value of 0.5 .

Figure S1. Fibroblast counts are similar in all conditions. Boxplots comparing average fibroblast counts per field of view for the low and high density hepatocyte conditions, both with the same amount of fibroblasts seeded. (A) The previous workflow displays no separation between the low and high density hepatocyte conditions $\left(Z^{\prime}\right.$-factor $=-12.39, p$-value $\left.=0.43\right)$. $(B)$ The new workflow also displays no separation between the low and high density hepatocyte conditions $\left(Z^{\prime}\right.$-factor $=-6.44$, $p$-value $\left.=0.20\right)$.

Figure S2. Nucleus size across conditions. (A) and (B) Fibroblast nuclei median diameter was slightly different between the old and new methods (median diameter old and new method $=$ 22.7 and 22.0 pixels, respectively; Wilcoxon rank sum test, $p<10^{-9}$ ). (C) and (D) Hepatocyte nuclei median diameter was also different between the old and new methods (median diameter old and new method $=16.2$ and 13.9 pixels, respectively; Wilcoxon rank sum test, $p<10^{-9}$ ). $(E)$ Data from (A-D) are displayed as boxplots to better compare the median values. Importantly, however, we note that the size differences shown are very sensitive to user-adjustable thresholding parameters which differ between the two methods, and these were not tuned to be the same; in other words, this difference in size could likely be eliminated through adjustment of parameters, if desired. All data are shown for the whole plate and not restricted to the highest and lowest hepatocyte count wells.

\section{Figure S3. DNA content (integrated intensity of DNA stain in the nucleus) across}

conditions. (A) and (B) Fibroblasts show distinct $2 \mathrm{~N}$ and $4 \mathrm{~N}$ peaks in integrated intensity, and have similar intensity between old and new methods. (C) and (D) Hepatocytes show a single integrated peak, indicating lack of cell proliferation, as expected. The lack of a right tail in (D) reflects a smaller threshold for hepatocyte segmentation in the new method. 


\section{Machine Learning}

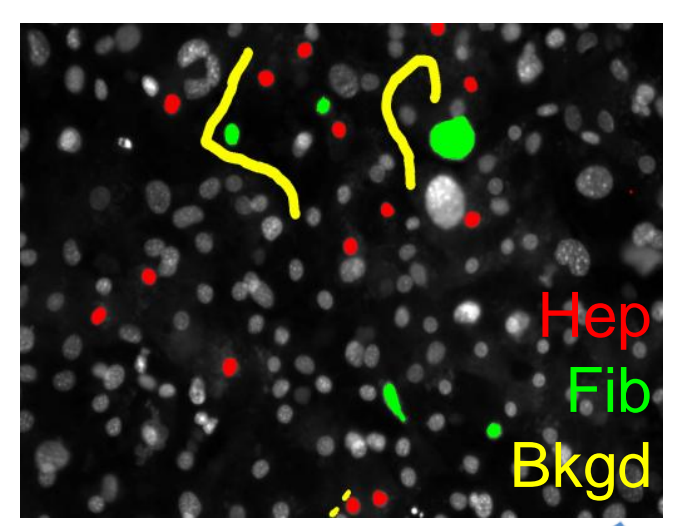

Iterate
Hepatocytes
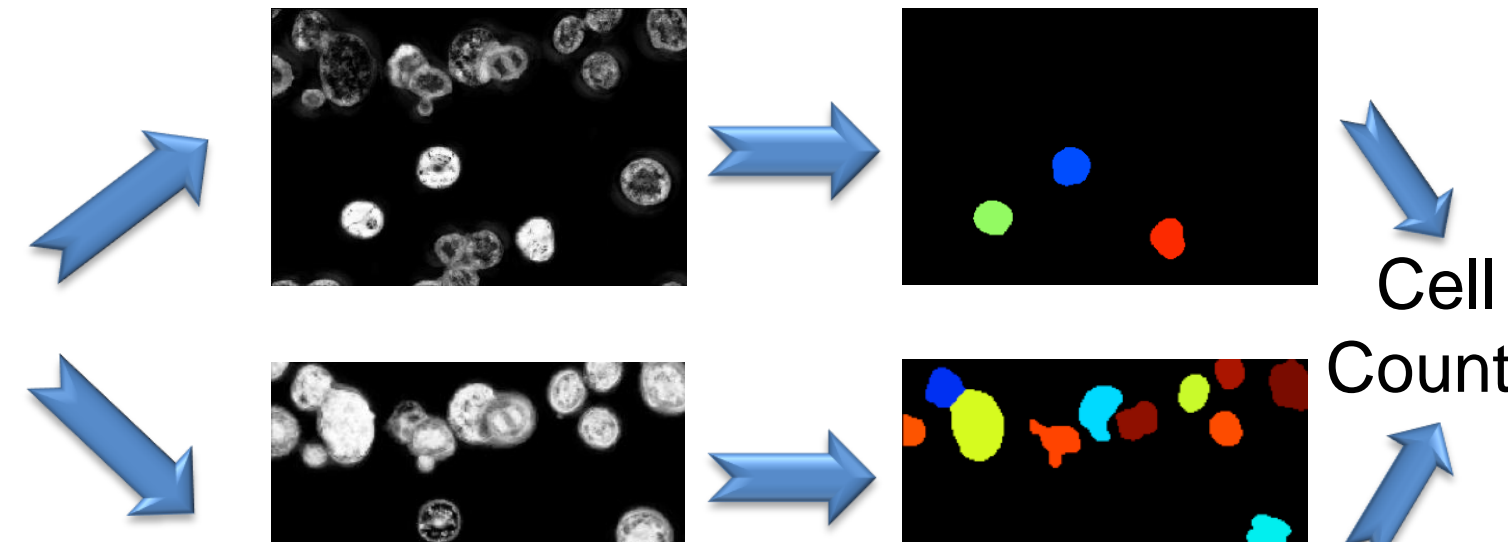

Counts

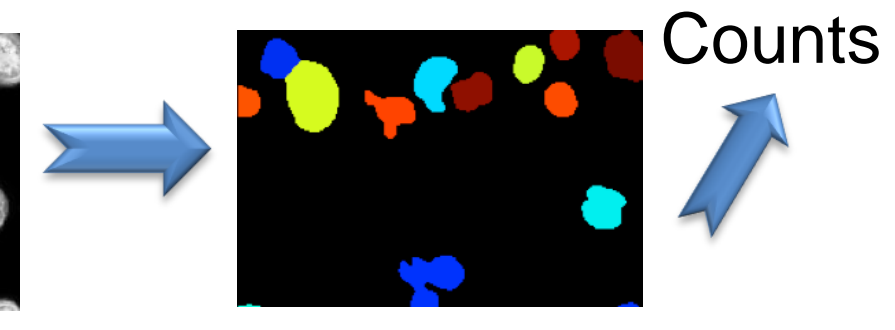

Fibroblasts 


\section{A. Previous Workflow}

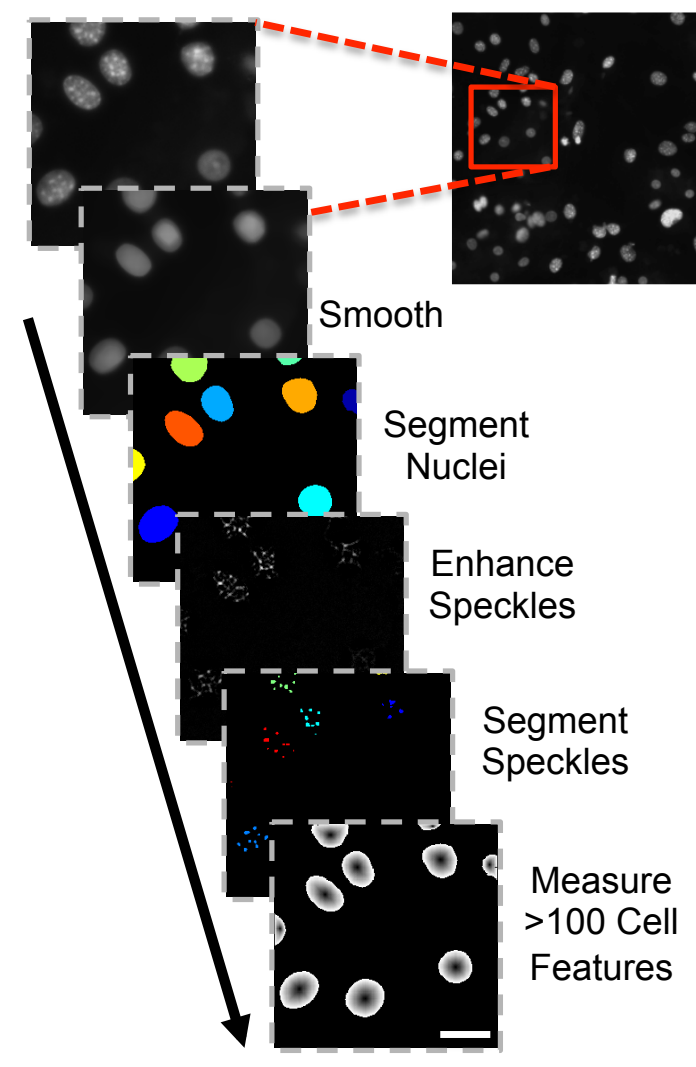

Create Training Set for Object-Based Machine Learning

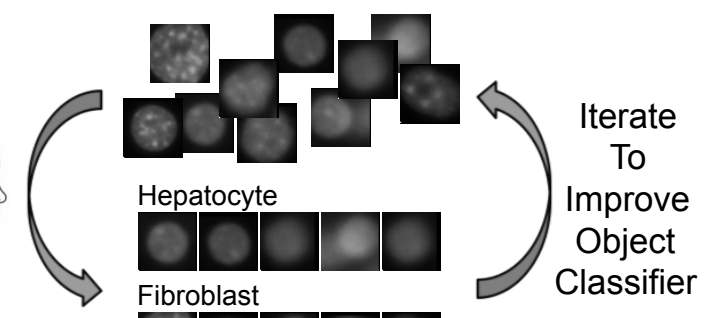

Fibroblast

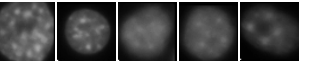

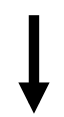

Cell Counts

\section{B. New Workflow}

Create Training Set for Pixel-Based Machine Learning

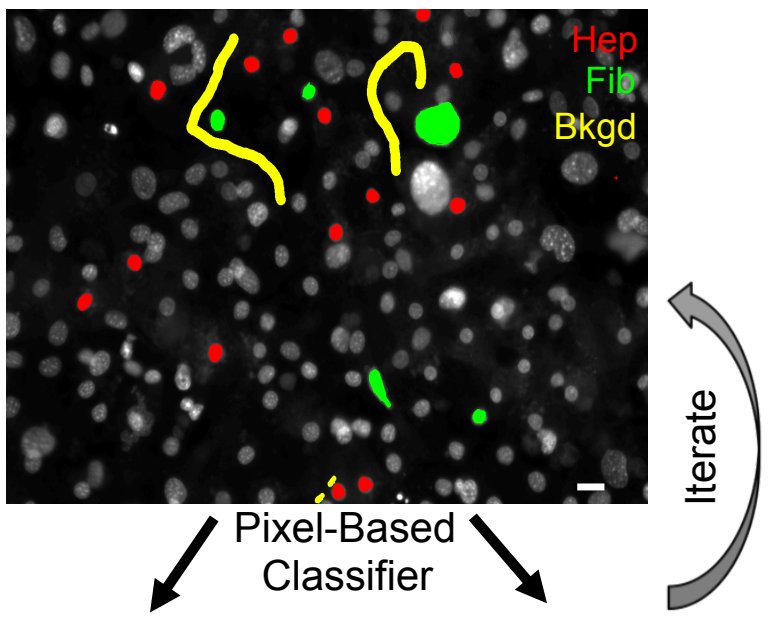

Hepatocyte

Fibroblast

Probability Map

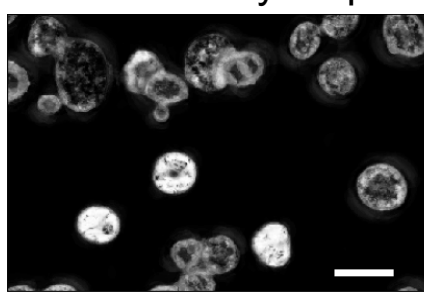

Probability Map

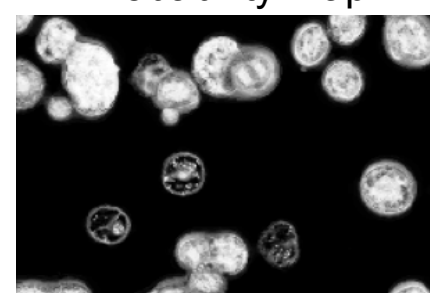

$$
\downarrow \text { Smooth \& Segment }
$$
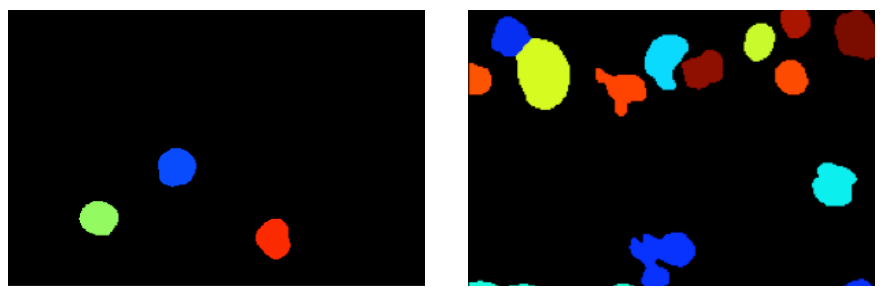

4

Cell Counts

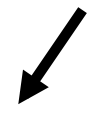


A. Raw Image

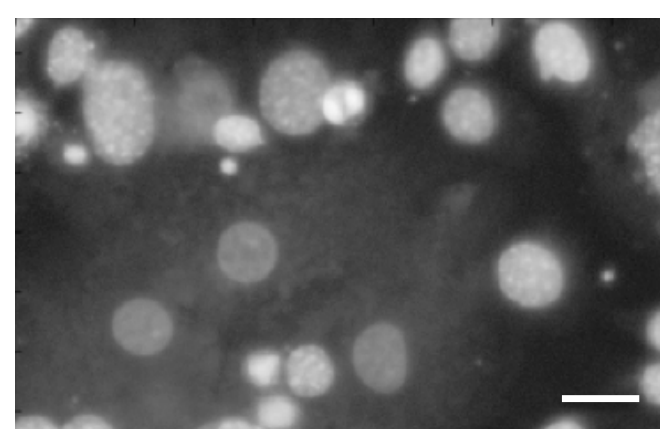

B. Previous Method (All Nuclei)

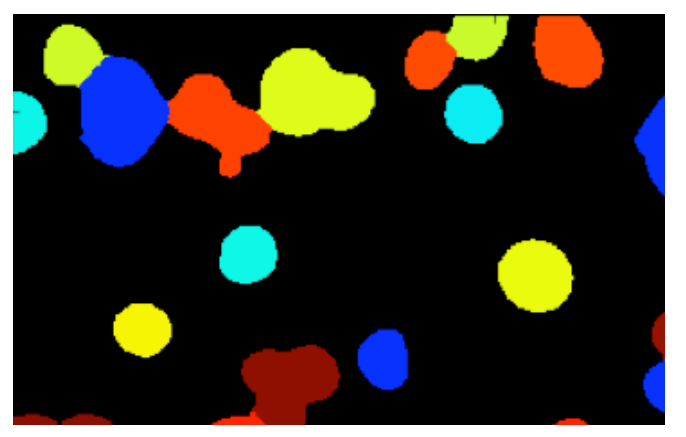

C. New Method (Hep)

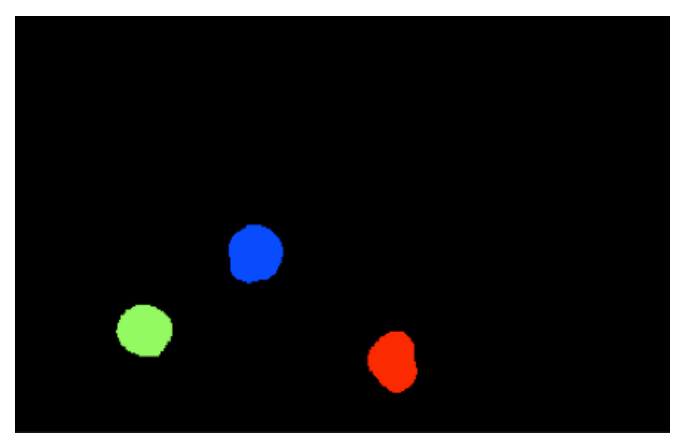

\section{New Method (Fib)}

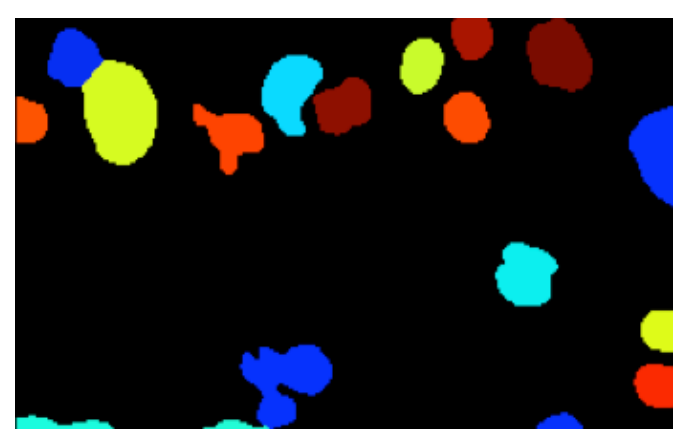


A. Previous Method

B. New Method
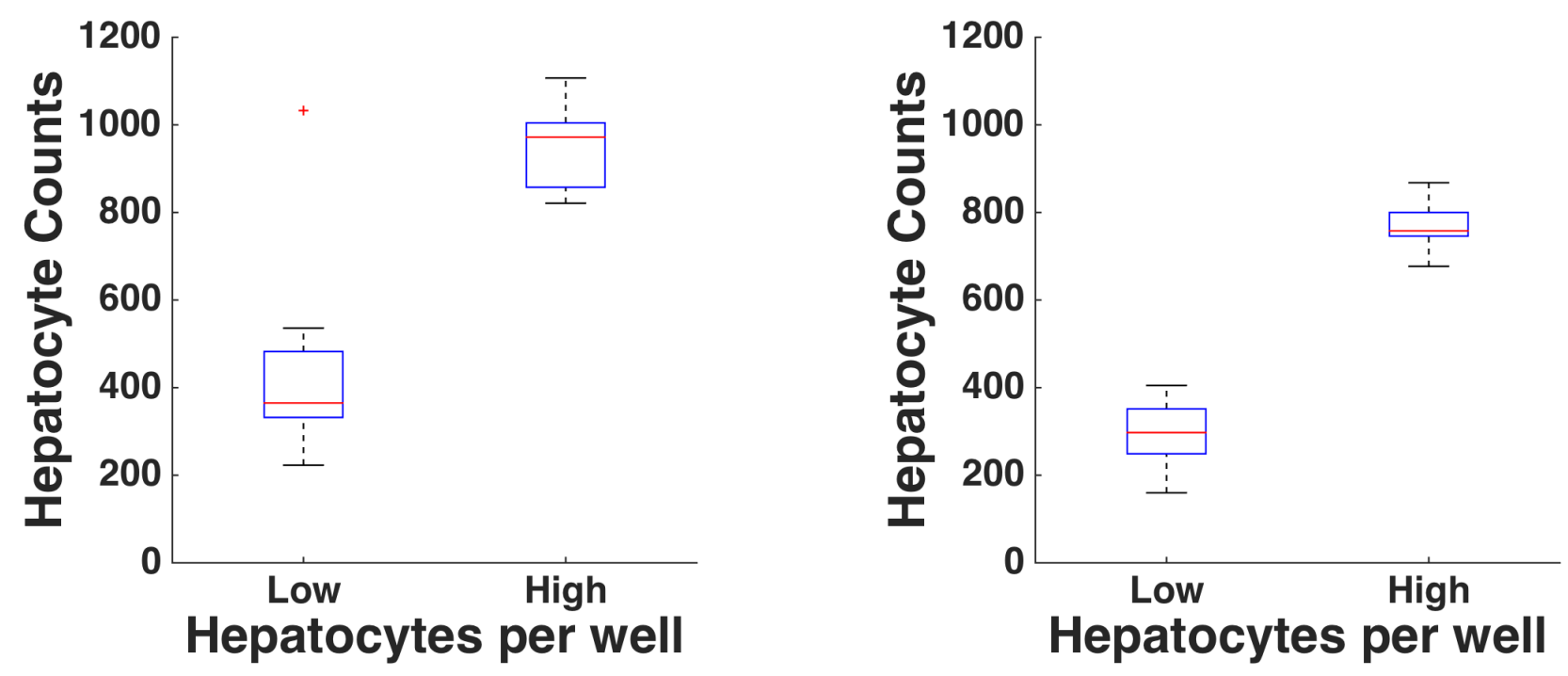